\title{
Presenters or Patients? A Crucial Distinction in Individual Health Assessments
}

\author{
G. Owen Schaefer ${ }^{1}$
}

Received: 6 October 2017 / Accepted: 22 February 2018

(C) National University of Singapore and Springer Nature Singapore Pte Ltd. 2018

\begin{abstract}
Individual health assessments (IHAs) for asymptomatic individuals provide a challenge to traditional distinctions between patient care and non-medical practice. They may involve undue radiation exposure, lead to false positives, and involve high out-of-pocket costs for recipients. A recent paper (Journal of the American College of Radiology 13(12): 1447-1457.e1, 2016) has criticised the use of IHAs and argued that recipients should be classified as 'presenters', not 'patients', to distinguish it from regular medical care. I critique this classificatory move, on two grounds: one, it is conceptually suspect. Two, it obviates the medical ethics framework for IHAs, potentially exposing recipients of IHAs to lower standards of oversight and protection. Responsible regulation of IHAs will be easier to ethically justify if those seeking IHAs are considered patients and not merely presenters.
\end{abstract}

Keywords Individual health assessments · Asymptomatic screening ·

Computed tomography $\cdot$ Medical language $\cdot$ Medical ethics

\section{Introduction and background}

The boundaries of medicine continue to shift, as patients become more empowered and traditional healthcare operations are taken over by new institutions and platforms. Selfdiagnosis by internet is now commonplace. Direct-to-consumer genetic testing companies bring Silicon Valley mindset to health assessments. Here, I will focus on screening programmes that have expanded beyond nationally sanctioned models towards a more consumer-demand based approach of 'individual health assessments'.

\footnotetext{
G. Owen Schaefer
}

medgos@nus.edu.sg

1 Centre for Biomedical Ethics, Yong Loo Lin School of Medicine, National University of Singapore, Singapore 
With this shift, some are tempted to say that recipients of such interventions are no longer patients. In this paper, I will argue we should resist such a reconceptualization. While new paradigms are certainly emerging, it is both conceptually sound and ethically important to retain the notion of 'patients' for those who undergo individual health assessments.

We can understand individual health assessments (IHAs) as diagnostic tests performed on healthy, asymptomatic individuals outside the context of a formal nationally sanctioned screening programme. This may take a variety of forms, but the most relevant for present context is CT scans (either whole body or targeted) on healthy, asymptomatic individuals without a particular family history prompting the test. The goal is to detect conditions (e.g. cancer) early and use follow-up care to prevent significant morbidity/mortality.

IHAs may at first blush seem unproblematic. Disease prevention is a laudable and traditional goal of medicine. IHAs can be cast as simply the next reasonable step in prognostication: whereas previously, external symptoms were our best cue for the onset of disease, internal imaging has added a powerful new tool in the ability of physicians to adequately predict and prevent disease. It provides more information about someone and is not more information always better?

However, a number of problems with IHAs have been noted in the literature. These include radiation exposure, false positives, overtreatment, and individual cost burdens.

\section{Radiation exposure}

Many IHAs, CT scans in particular, involve exposing recipients to some degree of radiation. Such exposure is well known to have the potential to cause harm. According to one estimate, a single full-body CT scan of a 45-year-old increases lifetime cancer mortality by .08\%; with 30 annual scans over a lifetime, that increased risk goes up to $1.9 \%$ (Brenner and Elliston 2004). The individual risk may seem marginal enough to not warrant particular scrutiny, but IHAs are better framed in terms of the lifetime risk. They are, after all, not designed as being responsive to particular symptoms. Rather, they may be marketed as part of a routine health check-ups.

Because IHAs are often packaged in this way, they run the serious risk of causing significant and excessive radiation exposure. For example, an analysis of radiation exposure in South Korea as a result of private-sector IHAs found between 3.6 and 24 times the annual dose limit (Lee 2016). And while South Korea has one of the higher international rates of IHA uptake, it is not necessarily exceptional-its use of CTs is comparable to that of Iceland and the USA (Kim 2016).

\section{False positives}

IHAs, like any screening test, run the risk of a Type 1 error: a seeming detection of disease that, upon further examination, turns out to be a false positive. False positives are particularly problematic for IHAs, which are by definition asymptomatic. The lack of symptoms for a particular disease increases the likelihood that detections of that disease are mistaken. So, for instance, lung cancer screenings via CT have been found to have a $21 \%$ false positive rate from even just 1 scan (Croswell 2010). This problem is only exacerbated for IHAs in individuals with no particular risk factors for a given disease. 
False positives have a number of harmful effects. The initial finding will mandate unnecessary further tests, which mean further radiation exposure and costs incurred. If further tests involve the same false positive error, it may also involve unindicated treatments, which carry their own risks and burdens. The same study cited above found $7 \%$ of false-positive reports resulted in unnecessary invasive treatment (Croswell 2010). And even if a false positive is invalidated before treatment begins, in the interim recipients may be subjected to undue anxiety, as individuals fear the onset of a disease and consider how it will negatively impact their lives and their families (Brewer et al. 2007).

\section{Overtreatment}

Even for true positives, a finding may result in overtreatment. Once an IHA detects and then verifies a finding - say, early cancer - preventative treatment may be indicated. Yet in many cases such treatment will be excessive because, even absent treatment, the disease would take too long to manifest itself and the individual would die from some other cause in the interim. Again, this is the result of IHAs being performed in asymptomatic individuals: the disease is present without symptoms, and it is often difficult to predict whether those symptoms will ever fully manifest.

One may object that this concern is overblown because, when a finding is verified, treatment will surely be guided by the best evidence. However, even some national screening programmes for asymptomatic individuals (only adopted after rigorous review of evidence) have had mixed results. In one of the most striking examples, a national rollout of thyroid cancer screening in South Korea led to a massive spike in diagnoses of thyroid cancer (up to 15-fold), but no concurrent substantial change in thyroid cancer mortality rates (Ahn et al. 2014). The risk is even greater for IHAs, which will generally have a weaker evidence base than national programmes.

\section{Cost}

In addition to the medical burdens of IHAs, such scans also require resources to perform. Due to poor evidence bases, IHAs are not typically paid for by health insurance schemes; instead, recipients pay for them out of pocket. As such, a private industry has arisen to sell IHAs directly to various populations (Lau and Illes 2009). That industry has come under criticism for exaggerated advertising claims, where downsides like the false positives and overtreatments mentioned above are downplayed and advantages exaggerated (Illes et al. 2004). This creates a problematic environment where consumers will spend non-trivial resources opt for IHAs as a result of being poorly informed.

\section{Recipients as 'presenters'}

In the context of these concerns, Malone et al. (2016) advocate reform and tighter standards surrounding the delivery of IHAs. Along the way, they advocate that recipients should be referred to as 'presenters' rather than 'patients'. Their reasoning 
is primarily conceptual, but it also alludes to a more substantive reason for the classification.

On the concept of 'patient', they write (Malone et al. 2016, 1448-49):

The prevailing health care model for radiology is one in which a patient, with symptoms or likelihood of disease, presents to a physician...A patient is a person who is a recipient of health care. In this context, a person on whom an examination is performed is the patient, as this term is commonly understood. However, an asymptomatic person presenting for a radiological procedure as part of IHA is frequently not a patient in this sense. For convenience, such persons are here referred to as the presenter.

The idea is that a 'patient' typically refers to someone for whom there is some indication for the healthcare being provided. IHAs by definition are in asymptomatic individuals and so they fall outside this ordinary usage of the term 'patient'. The term 'presenter' is offered as an alternative that is broader in scope, referring more generally to the fact that someone presents themselves for an IHA.

At the same time, there is also a concern that individuals seeking IHAs do not selfconceptualise as patients, due to being presumed healthy as well as the commercial nature of the tests mentioned above. The transition to 'patient' only occurs when there is a finding, and further treatment or testing is indicated.

Malone et al. also critique the idea that IHAs should be considered 'health care'. They say that an IHA for which there is no evidence base 'is not acceptable as a medical procedure in its present form" (Malone et al. 2016, 1453). To call a recipient of an IHA a 'patient', then, is in a sense to legitimise the IHA as healthcare that is reasonable to offer. By instead referring to IHA recipients as 'presenters', they can put pressure on their acceptability without begging the question in favour of IHAs.

\section{Objection 1: The Conceptual Problem}

Despite the above claims, we should accept that recipients of IHAs are indeed patients. Malone et al. rely on the WHO's definition of patient as a person who receives healthcare. Healthcare is in turn defined as "services received by individuals or communities to promote, maintain, monitor or restore health" (World Health Organization 2009, 22). This understanding makes no reference to a patient being symptomatic. Indeed, routine medical checkups involve healthy, asymptomatic individuals. Such individuals are typically referred to as the patients of their GPs; we do not say they only transition to being patients once care is received. This is because prevention of disease is one of the core goals of healthcare. IHAs, like routine medical checkups, share that similar aim of early detection of disease, and so to be consistent we should refer to both recipients as patients.

It could be insisted that IHAs are not like routine medical checkups, because routine medical checkups are widely accepted as indicated in the general population, whereas IHAs are not. However, we need not think that 'patient' 'is an honorific that automatically means any tests or treatments received are reasonable. If a physician due to negligence prescribes medicine wholly inappropriate for a patient's condition, we 
should not think they are thereby not practicing medicine or engaged in healthcare. Rather, they are practicing bad medicine; they are poorly serving their patients. Similarly, practitioners who offer IHAs without any good evidence base may be providing patients with poor healthcare- but they are still interacting with patients.

Alternatively, one could make an argument by analogy. The emergence of direct-toconsumer (DTC) health services has made it possible to receive personal medical information outside the traditional healthcare context. 23andme, for instance, offers genetic 'wellness' reports for the general population that include predisposition to certain conditions. But it does not make sense to call those engaging 23andme's services 'patients'. They are instead understood as customers or consumers (Annas and Elias 2014).

IHAs, like genetic tests, aim to provide the general asymptomatic population with personalised health information. So perhaps similar terminology should be used in both cases. This would militate in favour of 'consumer' language rather than 'presenter', but nevertheless would be a departure from traditional 'patient' language.

However, DTC genetic tests are a step further removed from traditional healthcare compared with IHAs. The whole process occurs remotely, with no personal interaction with a healthcare worker at any stage. IHAs, by contrast, are performed in person by a qualified specialist. While that specialist may be a radiographer, their direct presence creates a distinct context of interpersonal interaction and dependence that merits the categorisation of 'patient'.

\section{Objection 2: The Ethical Problem}

Despite IHAs falling within the scope of traditional definitions of patients, one may still insist that many recipients of IHAs do not see themselves as patients. As such, IHAs may fall in a category that warrants altering the WHO standard to conform to modern language usage.

If all that was at stake here were terminology, this rejoinder might have merit. However, 'patient' and 'presenter' are not of mere semantic difference. The term 'presenter' is meant to incorporate a more commercial, client-provider relationship than the term 'patient'. And as a matter of practice, there are significantly different ethical norms that govern general commercial relationships compared with patientphysician (or other healthcare provider) relationships.

First and foremost, physicians owe a fiduciary duty to their patients that is absent from most commercial relationships. The physician's fiduciary duty is to first and foremost act in the best interests of their patient. This is based on a level of trust required by patients, who typically lack the level of training and expertise needed to assess whether the physician's actions are sound (Chin 2001). By denying that recipients of IHAs are patients, we risk removing the fiduciary relationship between recipients and providers of IHAs. That could expose recipients to less responsible care, shifting the burden off providers to ensure IHAs are indeed indicated before offering them.

In addition, medical ethics more broadly has produced a significant set of frameworks that offer higher standards than what is expected in commercial transactions. The Principles approach identified four pillars: beneficence, non-maleficence, autonomy and justice. Autonomy may favour offering IHAs, insofar as fulfilling a patient request is 
responsive to their own stated preferences for healthcare. However, beneficence, nonmaleficence and justice militate against providing IHAs, for the reasons described above: they are of questionable benefit; have the risk of harming patients through radiation, false positives and overtreatment; and are potentially unjust, by requiring out-of-pocket expenditures by the patient who may have been swayed by attractive advertisements.

In the commercial realm, by contrast, autonomy takes precedence and there is more permissiveness. We do not ask car salespeople to only sell a unit if it is in the best interests of their customer; the bar is much more minimally that they should not deceive them. As long as the client agrees and is well-informed, there is not much reason to interfere.

This lower bar for a commercial framework does not mean no protections are warranted, of course. A 'presenter' standard could still justify restrictions on deceptive advertising from IHA providers. But this is much more minimal than the range of protections that a patient-centric approach could offer, which includes professional sanction, restriction on sales, and strong oversight of delivery. It also changes the ethical frame that would be brought to bear on public debates over policies regulating the practice. A medical frame will, appropriately, be more concerned with the potential indirect harms of a treatment caused by overtreatment and false positives.

\section{Conclusion}

We should call those receiving IHAs 'patients', not merely 'presenters'. This will help justify stronger protections against the downsides of IHAs. The free-market argument that those providing IHAs are simply responding to consumer demand will not fly in this framework - medical practitioners have a higher duty to ensure patients are not spending money on medically ill-advised, potentially harmful procedures.

This implication may seem purely semantic, focused on the terminology we use rather than the more important substantive debates over what level of regulation of IHAs is appropriate. However, the two are connected. How we describe and frame IHAs will significantly influence what governance mechanisms are deemed appropriate. It is therefore important that such framing is conceptually and ethically defensible.

Despite what has been said above, it might become the case that discussions of IHAs in light of Malone et al.'s work frame recipients of IHAs as presenters. In such circumstances, it will become incumbent upon interlocutors to emphasise that, despite the terminology, we should apply the higher standards of medical ethics to oversight of IHAs. The tests are employed by medical personnel, for diagnostic purposes, and should be governed as such.

\section{References}

Ahn, Hyeong Sik, Hyun Jung Kim, and H. Gilbert Welch. 2014. Korea's Thyroid-Cancer 'Epidemic'Screening and Overdiagnosis. New England Journal of Medicine 371 (19): 1765-1767. https://doi. org/10.1056/NEJMp1409841.

Annas, George J., and Sherman Elias. 2014. 23 andMe and the FDA. New England Journal of Medicine 370 (11): 985-988. https://doi.org/10.1056/NEJMp1316367.

Brenner, David J., and Carl D. Elliston. 2004. Estimated Radiation Risks Potentially Associated with FullBody CT Screening. Radiology 232 (3): 735-738. https://doi.org/10.1148/radiol.2323031095. 
Brewer, Noel T., Talya Salz, and Sarah E. Lillie. 2007. Systematic Review: The Long-Term Effects of FalsePositive Mammograms. Annals of Internal Medicine 146 (7): 502. https://doi.org/10.7326/0003-4819146-7-200704030-00006.

Chin, J.J. 2001. Doctor-Patient Relationship: A Covenant of Trust. Singapore Medical Journal 42 (12): 579581.

Croswell, Jennifer M. 2010. Cumulative Incidence of False-Positive Test Results in Lung Cancer Screening: A Randomized Trial. Annals of Internal Medicine 152 (8): 505. https://doi.org/10.7326/0003-4819-152-8201004200-00007.

Illes, Judy, Dylan Kann, Kim Karetsky, Phillip Letourneau, Thomas A. Raffin, Pamela Schraedley-Desmond, Barbara A. Koenig, and Scott W. Atlas. 2004. Advertising, Patient Decision Making, and Self-Referral for Computed Tomographic and Magnetic Resonance Imaging. Archives of Internal Medicine 164 (22): 2415. https://doi.org/10.1001/archinte.164.22.2415.

Kim, Yeonghee. 2016. Are We Being Exposed to Radiation in the Hospital? Environmental Health and Toxicology 31 (March): e2016005. https://doi.org/10.5620/eht.e2016005.

Lau, Patricia W., and Judy Illes. 2009. The Gray Zones of Privatized Imaging. The American Journal of Bioethics 9 (4): 21-22. https://doi.org/10.1080/15265160802716845.

Lee, Yun-Keun. 2016. Level of Radiation Dose in University Hospital Non-Insured Private Health Screening Programs in Korea. Environmental Health and Toxicology 31 (March): e2016007. https://doi.org/10.5620 /eht.e2016007.

Malone, Jim, Maria del Rosario Perez, Eva Godske Friberg, Mathias Prokop, Seung Eun Jung, Jurgen Griebel, and Steve Ebdon-Jackson. 2016. Justification of CT for Individual Health Assessment of Asymptomatic Persons: A World Health Organization Consultation. Journal of the American College of Radiology 13 (12): 1447-1457.e1. https://doi.org/10.1016/j.jacr.2016.07.020.

World Health Organization. 2009. Conceptual Framework for the International Classification for Patient Safety. http://www.who.int/patientsafety/implementation/taxonomy/icps_technical_report_en.pdf?ua=1. 